$\exists \int$ Journal of Logic \& Analysis 1:4 (2009) 1-23

ISSN 1759-9008

\title{
Decomposition of terms in Lucas sequences
}

\author{
ABDELMADJID BOUDAOUD
}

Let $P, Q$ be non-zero integers such that $D=P^{2}-4 Q$ is different from zero. The sequences of integers defined by

$$
\left\{\begin{array}{lll}
U_{n}=P U_{n-1}-Q U_{n-2} ; & U_{0}=0, & U_{1}=1 ; \\
V_{n}=P V_{n-1}-Q V_{n-2} ; & V_{0}=2, & V_{1}=P .
\end{array}\right.
$$

are called the Lucas sequences associated to the pair $(P, Q)$ [6,7]. In this paper we prove the following result: If $P, Q$ are such that $D$ is strictly positive, then, for unlimited $n$, each of the integers $U_{n}$ and $V_{n}$ differs by a limited integer from a product of two unlimited integers.

2000 Mathematics Subject Classification 11B39, 11A51, 26E35

Keywords: Lucas sequences, Fibonacci sequences, nonstandard analysis

\section{Introduction}

Let $N$ be any large integer. Proceeding directly to the factorization of $N$ is not an easy task, even unfeasible unless $N$ belongs to a particular family of integers. Then to surmount this major difficulty we might choose to ask about the factorization of an integer in a small neighborhood of $N$ instead of $N$. This is expressed through the following question: Is there a small integer $s$ such that $N=s+\mu \vartheta$, where $\mu$ and $\vartheta$ are two large integers?

The fact that the integer $N-s$ is a product of two large integers, gives an idea of its factorization. In the existing literature, the decomposition of integers is an immense problem which has been posed in several ways and treated by different methods (for example $[1,3,8])$.

This idea originated in [2], where we chose to work in the framework of nonstandard analysis $[4,5]$ to be able to give sense to the words "small", "large", ... and the question has the formulation: Is every unlimited integer the sum of a limited integer and a product of two unlimited integers ? To give a partial answer we provided some examples [2] and we devote the present work to another example concerning Lucas 
sequences. In the final section we give the classical equivalence of the result obtained and a general remark.

We start with a brief overview of Lucas sequences associated to a pair of integers [6]. Let $P, Q$ be non-zero integers. Consider the polynomial $p(x)=x^{2}-P x+Q$; its discriminant is $D=P^{2}-4 Q$ and the roots are

$$
\alpha=\frac{P+\sqrt{D}}{2}, \quad \beta=\frac{P-\sqrt{D}}{2} .
$$

Suppose that $P$ and $Q$ are such that $D \neq 0$. The sequences of integers

$$
\left\{\begin{array}{ccc}
U_{n}(P, Q)=\frac{\alpha^{n}-\beta^{n}}{\alpha-\beta} \quad \text { with } & U_{0}(P, Q)=0 \text { and } U_{1}(P, Q)=1 \\
V_{n}(P, Q)=\alpha^{n}+\beta^{n} & \text { with } & V_{0}(P, Q)=2 \text { and } V_{1}(P, Q)=P
\end{array}\right.
$$

are called the Lucas sequences associated to the pair $(P, Q)$. We will note by $U_{n}$ (resp. $\left.V_{n}\right)$ the element $U_{n}(P, Q)$ (resp. $\left.V_{n}(P, Q)\right)$.

It can be proved that for $n \geq 2$

$$
\begin{array}{lll}
U_{n}=P U_{n-1}-Q U_{n-2} ; & U_{0}=0, & U_{1}=1, \\
V_{n}=P V_{n-1}-Q V_{n-2} ; & V_{0}=2, & V_{1}=P .
\end{array}
$$

In the particular case $(P, Q)=(1,-1)$, the sequence $\left(U_{n}\right)_{n \geq 0}$ begins $0,1,1,2,3,5,8,13$ $\ldots$ and was first considered by Fibonacci. The companion sequence of the Fibonacci numbers, still with $(P, Q)=(1,-1)$, is the sequence of Lucas numbers $\left(V_{n}\right)_{n \geq 0}$ and it begins $2,1,3,4,7,11,18 \ldots$.

We give here some known results $[6,7]$

$$
V_{2 n}=\left(V_{n}\right)^{2}-2 Q^{n} \text {. }
$$

Let $p$ be a prime integer, then

$$
\left\{\begin{array}{cc}
U_{p}=\left(\frac{D}{p}\right) \bmod (p) & \text { for } p \geq 3 \\
V_{p}=P \bmod (p) & \text { for } p \geq 2
\end{array}\right.
$$

where $\left(\frac{D}{p}\right)$ represents the Legendre symbol which is, according to the relation between $p$ and $D$, one of the values $-1,0,+1$. In addition, if $n, k \geq 1$, then

$$
U_{n} \mid U_{n k} \text { for all } k, \quad V_{n} \mid V_{n k} \text { if } k \text { is odd. }
$$

Moreover

$$
\left\{\begin{array}{l}
U_{n}(-P, Q)=(-1)^{n-1} U_{n}(P, Q), \\
V_{n}(-P, Q)=(-1)^{n} V_{n}(P, Q) .
\end{array}\right.
$$


Fermat's Little Theorem. If $p$ is a prime number and if $a$ is an integer, then

$$
a^{p} \equiv a[p] .
$$

In particular, if $p$ does not divide a then $a^{p-1} \equiv 1[p]$.

External recurrence principle [4]. For all internal or external formula $F(n)$, we have

$$
\left[F(0) \quad \text { and } \quad \forall^{s t} n(F(n) \Longrightarrow F(n+1))\right] \Longrightarrow \forall^{s t} n F(n) \text {. }
$$

Notations. Let $x, y$ be real numbers (not necessarily integers) .

(i) $x \approx 0$ ( resp. $x \approx+\infty$ ) denotes that $x$ is infinitesimal ( resp. $x$ is positive unlimited). We have an analogous definition for $x \approx-\infty$.

(ii) $x$ and $y$ are called infinitely close, denoted by $x \approx y$, if $x-y \approx 0$.

(iii) We say that $x$ is appreciable if it is neither unlimited nor infinitesimal.

(iv) The inequality $x \gtrsim y$ means that $x>y$ and $x \not y$. We have an analogous definition for $\succsim$.

(v) The Greek letter $\phi$ is used for an infinitesimal strictly positive. Two occurrences of $\phi$ are not necessarily equal.

(vi) $\mathbb{P}$ represents the set of all prime integers.

\section{Main result}

Theorem 2.1 If $P, Q$ are such that $D>0$, then, for unlimited $n$, each of the integers $U_{n}$ and $V_{n}$ differs by a limited integer from a product of two unlimited integers.

Let $P$ and $Q$ be such that $D>0$ and let $n \approx+\infty$. We put $\lambda=\frac{P}{\sqrt{D}}$. In order to prove the main result we need the following lemmas.

Lemma 2.2 (a) $\alpha \neq \beta, \max (|\alpha|,|\beta|) \gtrsim 1$ and

$$
\frac{\beta}{\alpha}=\frac{\lambda-1}{\lambda+1} .
$$

(b) If $P>0$, then

(i) $|\alpha|>|\beta|$,

(ii) $1-\frac{\beta}{\alpha}$ is positive infinitesimal $\Longleftrightarrow \lambda \approx+\infty$, 
(iii) $1+\frac{\beta}{\alpha}$ is positive infinitesimal $\Longleftrightarrow \lambda$ is positive infinitesimal,

(iv) $\frac{\beta}{\alpha} \not \approx \pm 1$ if and only if $\lambda$ is appreciable and positive.

(c) If $P<0$,

(i) $|\alpha|<|\beta|$,

(ii) $1-\frac{\alpha}{\beta}$ is positive infinitesimal $\Longleftrightarrow \lambda \approx-\infty$,

(iii) $1+\frac{\alpha}{\beta}$ is positive infinitesimal $\Longleftrightarrow \lambda$ is negative infinitesimal,

(iv) $\frac{\alpha}{\beta} \not \pm \pm 1$ if and only if $\lambda$ is appreciable and negative.

Proof (a) $\alpha \neq \beta$ because $\alpha=\frac{P+\sqrt{D}}{2}$ and $\beta=\frac{P-\sqrt{D}}{2}$. The following are the possible cases.

(i) $P>1$. In this case $\alpha \gtrsim 1$.

(ii) $P=1$. In this case $Q$ must be strictly negative and therefore $\alpha \gtrsim 1$.

(iii) $P=-1$. In this case $Q$ must be strictly negative and therefore $|\beta| \gtrsim 1$.

(iv) $P<-1$. In this case $|\beta| \gtrsim 1$.

Hence

$$
\max (|\alpha|,|\beta|) \gtrsim 1 \text {. }
$$

By (1.1), $\frac{\beta}{\alpha}=\frac{\lambda-1}{\lambda+1}$.

(b) If $P>0$, then it is immediate that $|\alpha|>|\beta|$. Furthermore, $\lambda>0$ and the remainder of the proof can be deduced from the graph of the function $\frac{\beta}{\alpha}(\lambda)=\frac{\lambda-1}{\lambda+1}$ which is strictly increasing from $\left[0,+\infty\left[\right.\right.$ onto [-1, 1 [, where $\lim _{\lambda \longrightarrow 0^{+}} \frac{\beta}{\alpha}(\lambda)=-1$ and $\lim _{\lambda \longrightarrow+\infty} \frac{\beta}{\alpha}(\lambda)=1$.

(c) This is similar to (b).

Remark. By (1-7) we need to prove the following lemmas only for $P>0$ in which case $\alpha$ is positive and according to Lemma $2.2 \alpha>|\beta|$. Consequently $\alpha \gtrsim 1$.

Lemma 2.3 Each of $\left|U_{n}\right|$ and $\left|V_{n}\right|$ is of the form $\omega n$, where $\omega$ is unlimited ( $\omega$ is not necessarily the same in each case). 
Proof By (1-2)

$$
\left\{\begin{aligned}
U_{n} & =\alpha^{n-1}\left(\frac{1-(\beta / \alpha)^{n}}{1-(\beta / \alpha)}\right) \\
V_{n} & =\alpha^{n}\left(1+(\beta / \alpha)^{n}\right)
\end{aligned}\right.
$$

We divide the proof into three cases.

(A) $\frac{\beta}{\alpha}=1-\phi$. Then $0<(\beta / \alpha)^{n}<\beta / \alpha<1$. Hence $1-(\beta / \alpha)^{n}>1-(\beta / \alpha)>0$. By (2-2) it follows $U_{n}=\alpha^{n-1} c$ with $c>1$ and therefore $U_{n}=\omega n$, where $\omega \approx+\infty$. Also $V_{n}=\alpha^{n} d$ with $d>1$ and consequently $V_{n}=\omega n$, where $\omega \approx+\infty$.

(B) $\frac{\beta}{\alpha}=-1+\phi$. According to Lemma 2.2, $\lambda$ is positive infinitesimal. There are two subcases.

(1) $n$ is odd. By (2-2)

$$
\begin{aligned}
U_{n} & =\alpha^{n-1}\left(\frac{1-(-1+\phi)^{n}}{1-(-1+\phi)}\right)=\alpha^{n-1} \frac{\left(1+(1-\phi)^{n}\right)}{2-\phi} \\
& =\alpha^{n-1} a
\end{aligned}
$$

where $a$ is appreciable and positive. Therefore $U_{n}=\omega n$, where $\omega \approx+\infty$. Concerning $V_{n}$ by (2-1) and (2-2), $V_{n}=\alpha^{n}\left(1+\left(\frac{\lambda-1}{\lambda+1}\right)^{n}\right)$. Hence

$$
V_{n}=\alpha^{n}\left(1-\left(\frac{1-\lambda}{\lambda+1}\right)^{n}\right)>\alpha^{n}\left(1-\frac{1}{(\lambda+1)^{n}}\right)
$$

and then $V_{n}>\alpha^{n} \frac{n \lambda}{(\lambda+1)^{n}}$ which implies $\frac{V_{n}}{n}>\alpha^{n} \frac{\lambda}{(\lambda+1)^{n}}$. Moreover $\frac{\lambda}{(\lambda+1)^{n}} \alpha^{n} \approx$ $+\infty$, indeed replacing $\alpha$ by $\frac{P+\sqrt{D}}{2}$ we get

$$
\begin{aligned}
\frac{\lambda}{(\lambda+1)^{n}} \alpha^{n} & =\frac{\lambda}{(\lambda+1)^{n}}(1+\lambda)^{n}\left(\frac{\sqrt{D}}{2}\right)^{n} \\
& =\frac{P}{2}\left(\frac{\sqrt{D}}{2}\right)^{n-1},
\end{aligned}
$$

where $\frac{P}{2}\left(\frac{\sqrt{D}}{2}\right)^{n-1} \approx+\infty$ because $\sqrt{D} \approx+\infty$. Therefore $V_{n}$ is of the form $\omega n$, where $\omega \approx+\infty$. 
(2) $n$ is even. By (2-2)

$$
\begin{aligned}
U_{n}=\alpha^{n-1}\left(\frac{1-(\beta / \alpha)^{n}}{1-(\beta / \alpha)}\right) & =\frac{\alpha^{n-1}}{2-\phi}\left(1-(\beta / \alpha)^{n}\right) \\
& =\frac{\alpha^{n-1}}{a}\left(1-(\beta / \alpha)^{n}\right),
\end{aligned}
$$

where $a=2-\phi$ is appreciable. By (2-1), $\frac{\beta}{\alpha}=\frac{\lambda-1}{\lambda+1}$ where $\lambda$ is positive infinitesimal, and since $n$ is even,

$$
U_{n}=\frac{\alpha^{n-1}}{a}\left(1-\left(\frac{1-\lambda}{\lambda+1}\right)^{n}\right)
$$

From $\frac{1}{(\lambda+1)^{n}}>\left(\frac{1-\lambda}{\lambda+1}\right)^{n}$ it follows that $0<1-\frac{1}{(\lambda+1)^{n}}<1-\left(\frac{1-\lambda}{\lambda+1}\right)^{n}$, hence $U_{n}>\frac{\alpha^{n-1}}{a}\left(1-\frac{1}{(\lambda+1)^{n}}\right)$ and then $U_{n}>\frac{\alpha^{n-1}}{a} \frac{n \lambda}{(\lambda+1)^{n}}$. So

$$
\frac{U_{n}}{n}>\frac{\alpha^{n-1}}{a} \frac{\lambda}{(\lambda+1)^{n}} .
$$

Replacing $\alpha$ (resp. $\lambda$ ) by $\frac{P+\sqrt{D}}{2}$ (resp. $\frac{P}{\sqrt{D}}$ ), we get

$$
\frac{\alpha^{n-1}}{a} \frac{\lambda}{(\lambda+1)^{n}}=\frac{(\sqrt{D})^{n-2} P}{2^{n-1} a(1+\lambda)} \text {. }
$$

Finally, from $D \approx+\infty, \lambda \approx 0$ and $a$ is appreciable we obtain $\frac{\alpha^{n-1}}{a} \frac{\lambda}{(\lambda+1)^{n}} \approx+\infty$ and it follows that $\frac{U_{n}}{n} \approx+\infty$. Therefore $U_{n}=\omega n$, where $\omega \approx+\infty$.

Concerning $V_{n}$, the fact that

$$
V_{n}=\alpha^{n}\left(1+\left(\frac{\beta}{\alpha}\right)^{n}\right)=\alpha^{n}\left(1+(-1+\phi)^{n}\right)
$$

implies $V_{n}=\omega n$ with $\omega \approx+\infty$ because $\left(1+(-1+\phi)^{n}\right)>1$.

(C) $\frac{\beta}{\alpha} \not \approx \pm 1$ : By (2-2), $U_{n}=\alpha^{n-1}\left(\frac{1-(\beta / \alpha)^{n}}{1-(\beta / \alpha)}\right)$ hence $U_{n}=\alpha^{n-1} a$, where $a$ is appreciable. Therefore $U_{n}=\omega n$, where $\omega \approx+\infty . V_{n}=\alpha^{n}\left(1+\left(\frac{\beta}{\alpha}\right)^{n}\right)=$ $\alpha^{n} a$ where $a$ is appreciable, therefore $V_{n}=\omega n$ with $\omega \approx+\infty$, and the proof is complete. 
Lemma 2.4 If $n$ is of the form $n_{1} n_{2}$ with $n_{1}>1$ and $n_{2}>1$, then $\frac{\left|U_{n_{1} n_{2}}\right|}{\left|U_{n_{1}}\right|}$ and $\frac{\left|V_{n_{1} n_{2}}\right|}{\left|V_{n_{1}}\right|}$ are unlimited.

Proof Since $n \approx+\infty$, at least one of $n_{1}$ and $n_{2}$ is unlimited. By (1-2)

$$
\left\{\begin{aligned}
\frac{U_{n_{1} n_{2}}}{U_{n_{1}}} & =\frac{\alpha^{n_{1} n_{2}}}{\alpha^{n_{1}}}\left(\frac{1-(\beta / \alpha)^{n_{1} n_{2}}}{1-(\beta / \alpha)^{n_{1}}}\right), \\
\frac{V_{n_{1} n_{2}}}{V_{n_{1}}} & =\frac{\alpha^{n_{1} n_{2}}}{\alpha^{n_{1}}}\left(\frac{1+(\beta / \alpha)^{n_{1} n_{2}}}{1+(\beta / \alpha)^{n_{1}}}\right) .
\end{aligned}\right.
$$

We have three cases.

(A) $\frac{\beta}{\alpha}=1-\phi$. By (2-3) and the fact that $1-\left(\frac{\beta}{\alpha}\right)^{n_{1} n_{2}}>1-\left(\frac{\beta}{\alpha}\right)^{n_{1}}>0$ we have

$$
\frac{U_{n_{1} n_{2}}}{U_{n_{1}}}=\alpha^{n_{1}\left(n_{2}-1\right)} c=\alpha^{n-n_{1}} c,
$$

where $c>1$. Since $\left(n-n_{1}\right) \approx+\infty$ then $\frac{U_{n_{1} n_{2}}}{U_{n_{1}}} \approx+\infty$. Also by $(2-3), \frac{V_{n_{1} n_{2}}}{V_{n_{1}}}=$ $\alpha^{n_{1}\left(n_{2}-1\right)} c=\alpha^{n-n_{1}} c$, where $c$ is positive and appreciable. From the fact that $\left(n-n_{1}\right) \approx$ $+\infty$ we have $\frac{V_{n_{1} n_{2}}}{V_{n_{1}}} \approx+\infty$.

(B) $\frac{\beta}{\alpha}=-1+\phi$. By Lemma 2.2, $\lambda$ is positive infinitesimal. There are three subcases.

(1) $n_{1}$ is even. Then $n_{1} n_{2}$ is even and $\frac{U_{n_{1} n_{2}}}{U_{n_{1}}}=\alpha^{n_{1}\left(n_{2}-1\right)} c$ with $c>1$ because $1-\left(\frac{\beta}{\alpha}\right)^{n_{1} n_{2}}>1-\left(\frac{\beta}{\alpha}\right)^{n_{1}}>0$. Hence $\frac{U_{n_{1} n_{2}}}{U_{n_{1}}} \approx+\infty$. Concerning $V_{n}$ we have $\frac{V_{n_{1} n_{2}}}{V_{n_{1}}}=\frac{\alpha^{n_{1} n_{2}}}{\alpha^{n_{1}}}\left(\frac{1+(\beta / \alpha)^{n_{1} n_{2}}}{1+(\beta / \alpha)^{n_{1}}}\right)$. Since $n_{1}$ and $n_{1} n_{2}$ are even and $-1<\beta / \alpha<0$, then

$$
\begin{aligned}
\frac{V_{n_{1} n_{2}}}{V_{n_{1}}} & =\frac{\alpha^{n_{1} n_{2}}}{\alpha^{n_{1}}} c \\
& =\alpha^{n_{1}\left(n_{2}-1\right)} c \approx+\infty
\end{aligned}
$$

because $c$ is positive and appreciable and $\alpha^{n_{1}\left(n_{2}-1\right)} \approx+\infty$.

(2) $n_{1}$ and $n_{2}$ are both odd. From $\frac{U_{n_{1} n_{2}}}{U_{n_{1}}}=\alpha^{n_{1}\left(n_{2}-1\right)} \frac{\left(1-(-1+\phi)^{n_{1} n_{2}}\right)}{\left(1-(-1+\phi)^{n_{1}}\right)}$ we have

$$
\begin{aligned}
\frac{U_{n_{1} n_{2}}}{U_{n_{1}}} & =\alpha^{n_{1}\left(n_{2}-1\right)} \frac{\left(1+(1-\phi)^{n_{1} n_{2}}\right)}{\left(1+(1-\phi)^{n_{1}}\right)} \\
& =\alpha^{n_{1}\left(n_{2}-1\right)} a \approx+\infty
\end{aligned}
$$


because $a$ is positive and appreciable. Also

$$
\begin{aligned}
\frac{V_{n_{1} n_{2}}}{V_{n_{1}}} & =\alpha^{n_{1}\left(n_{2}-1\right)} \frac{\left(1+(-1+\phi)^{n_{1} n_{2}}\right)}{\left(1+(-1+\phi)^{n_{1}}\right)} \\
& =\alpha^{n_{1}\left(n_{2}-1\right)} \frac{\left(1-(1-\phi)^{n_{1} n_{2}}\right)}{\left(1-(1-\phi)^{n_{1}}\right)}
\end{aligned}
$$

Since $1-(1-\phi)^{n_{1} n_{2}}>1-(1-\phi)^{n_{1}}>0$, then $\frac{V_{n_{1} n_{2}}}{V_{n_{1}}}=\alpha^{n_{1}\left(n_{2}-1\right)} c$ with $c \geq 1$ which implies $\frac{V_{n_{1} n_{2}}}{V_{n_{1}}} \approx+\infty$ because $\alpha^{n_{1}\left(n_{2}-1\right)} \approx+\infty$.

(3) $n_{1}$ is odd, $n_{2}$ is even. Then $\frac{U_{n_{1} n_{2}}}{U_{n_{1}}}=\frac{\alpha^{n_{1}\left(n_{2}-1\right)}}{a}\left(1-\left(\frac{\beta}{\alpha}\right)^{n_{1} n_{2}}\right)$, where $a=\left(1-\left(\frac{\beta}{\alpha}\right)^{n_{1}}\right)$ is appreciable and strictly positive because $-1<\left(\frac{\beta}{\alpha}\right)^{n_{1}}<0$. Since $\lambda$ is positive infinitesimal and $\frac{\beta}{\alpha}=\frac{\lambda-1}{\lambda+1}$ then

$$
\frac{U_{n_{1} n_{2}}}{U_{n_{1}}}=\frac{\alpha^{n_{1}\left(n_{2}-1\right)}}{a}\left(1-\left(\frac{1-\lambda}{1+\lambda}\right)^{n_{1} n_{2}}\right) .
$$

Now

$$
\begin{aligned}
\frac{\alpha^{n_{1}\left(n_{2}-1\right)}}{a}\left(1-\left(\frac{1-\lambda}{1+\lambda}\right)^{n_{1} n_{2}}\right) & >\frac{\alpha^{n_{1}\left(n_{2}-1\right)}}{a}\left(1-\frac{1}{(1+\lambda)^{n_{1} n_{2}}}\right) \\
& >\frac{\alpha^{n_{1}\left(n_{2}-1\right)}}{a} \frac{n_{1} n_{2} \lambda}{(1+\lambda)^{n_{1} n_{2}}} .
\end{aligned}
$$

Replacing $\lambda$ by $\frac{P}{\sqrt{D}}$, we get

$$
\frac{\alpha^{n_{1}\left(n_{2}-1\right)}}{a} \frac{n_{1} n_{2} \lambda}{(1+\lambda)^{n_{1} n_{2}}}=\frac{n_{1} n_{2} P(\sqrt{D})^{n_{1} n_{2}-1}}{\alpha^{n_{1}} a \cdot 2^{n_{1} n_{2}}}
$$

which implies

$$
\frac{\alpha^{n_{1}\left(n_{2}-1\right)}}{a} \frac{n_{1} n_{2} \lambda}{(1+\lambda)^{n_{1} n_{2}}} \geq \frac{n_{1} n_{2} P(\sqrt{D})^{n_{1} n_{2}-n_{1}-1}}{a \cdot 2^{n_{1} n_{2}}}
$$

because $\alpha<\sqrt{D}$. Since $\sqrt{D}>2^{3}$,

$$
\frac{n_{1} n_{2} P(\sqrt{D})^{n_{1} n_{2}-n_{1}-1}}{a \cdot 2^{n_{1} n_{2}}} \geq \frac{n_{1} n_{2} P .2^{n_{1}\left(2 n_{2}-3\right)-3}}{a} .
$$

Therefore

$$
\frac{\alpha^{n_{1}\left(n_{2}-1\right)}}{a} \frac{n_{1} n_{2} \lambda}{(1+\lambda)^{n_{1} n_{2}}} \geq \frac{n_{1} n_{2} P \cdot 2^{n_{1}\left(2 n_{2}-3\right)-3}}{a} \approx+\infty
$$


and then $\frac{U_{n_{1} n_{2}}}{U_{n_{1}}} \approx+\infty$.

Concerning $\frac{V_{n_{1} n_{2}}}{V_{n_{1}}}$, we have $\frac{V_{n_{1} n_{2}}}{V_{n_{1}}}=\alpha^{n_{1}\left(n_{2}-1\right)} \frac{\left(1+(1-\phi)^{n_{1} n_{2}}\right)}{\left(1-(1-\phi)^{n_{1}}\right)}$. The facts that $1+$ $(1-\phi)^{n_{1} n_{2}}$ is appreciable, $\left.1-(1-\phi)^{n_{1}} \in\right] 0,1\left[\right.$ and $\alpha^{n_{1}\left(n_{2}-1\right)} \approx+\infty$ lead to $\frac{V_{n_{1} n_{2}}}{V_{n_{1}}} \approx$ $+\infty$.

(C) $\frac{\beta}{\alpha} \not \approx \pm 1$. The fact that $\frac{U_{n_{1} n_{2}}}{U_{n_{1}}}=\alpha^{n_{1}\left(n_{2}-1\right)} a, \frac{V_{n_{1} n_{2}}}{V_{n_{1}}}=\alpha^{n_{1}\left(n_{2}-1\right)} b$, where $\alpha^{n_{1}\left(n_{2}-1\right)} \approx+\infty, a$ and $b$ are appreciable and positive mean that $\frac{U_{n_{1} n_{2}}}{U_{n_{1}}} \approx+\infty$ and $\frac{V_{n_{1} n_{2}}}{V_{n_{1}}} \approx+\infty$ which finishes the proof.

Lemma 2.5 For every $i \geq 2,\left|U_{i}\right|<\left|U_{i+1}\right| \quad \& \quad\left|V_{i}\right|<\left|V_{i+1}\right|$.

Proof Let $i \geq 2$. We have three cases.

(A) $\frac{\beta}{\alpha}=1-\phi$. By Lemma 2.2, $\lambda \approx+\infty$ and then $\alpha \approx+\infty$. Now $\frac{U_{i+1}}{U_{i}}=$ $\frac{\alpha^{i+1}-\beta^{i+1}}{\alpha^{i}-\beta^{i}}=\alpha \frac{\left(1-r^{i+1}\right)}{\left(1-r^{i}\right)}$, where $r=\frac{\beta}{\alpha}=1-\phi$. Since $1-r^{i+1}>1-r^{i}>0$ and $\alpha \approx+\infty$, then $\frac{U_{i+1}}{U_{i}}>1$. Also we have $\frac{V_{i+1}}{V_{i}}=\alpha \frac{\left(1+r^{i+1}\right)}{\left(1+r^{i}\right)}$. Since $\frac{\left(1+r^{i+1}\right)}{\left(1+r^{i}\right)}$ is appreciable and $\alpha \approx+\infty$, then $\frac{V_{i+1}}{V_{i}} \approx+\infty$. That is, $\frac{V_{i+1}}{V_{i}}>1$.

(B) $\frac{\beta}{\alpha}=-1+\phi$. By Lemma $2.2, \lambda$ is positive infinitesimal. Since $P$ is supposed positive and $\lambda$ is positive infinitesimal, we directly get $Q \approx-\infty$. Therefore, from $Q<0$, we obtain $0<U_{2}<U_{3}<U_{4}<\ldots$. Similarly, $0<V_{2}<V_{3}<V_{4}<\ldots$.

(C) $\frac{\beta}{\alpha} \not \pm \pm 1$. According to Lemma 2.2, $\lambda$ is appreciable and strictly positive. $\frac{U_{i+1}}{U_{i}}=\alpha \frac{\left(1-r^{i+1}\right)}{\left(1-r^{i}\right)}$ with $r=\frac{\beta}{\alpha}=\frac{\lambda-1}{\lambda+1}$, where $\lambda$ is different from 1 because otherwise $Q=0$. We divide the rest of the proof into the following cases.

(1) $\lambda \in] 0,1\left[\right.$. Then $0<\frac{P}{\sqrt{P^{2}-4 Q}}<1$ and consequently $Q<0$. Hence, as in the case B) of this lemma, we have $0<U_{2}<U_{3}<U_{4}<\ldots$ and $0<V_{2}<V_{3}<$ $V_{4}<\ldots$. 
(2) $\lambda>1$. Then $r$ is appreciable and positive and $r<1$. Since $\frac{U_{i+1}}{U_{i}}=$ $\alpha \frac{\left(1-r^{i+1}\right)}{\left(1-r^{i}\right)}$ and $1-r^{i+1}>1-r^{i}>0$, then $\frac{U_{i+1}}{U_{i}}>\alpha>1$. Concerning $\frac{V_{i+1}}{V_{i}}$ the fact that $\lambda=\frac{P}{\sqrt{P^{2}-4 Q}}>1$ implies $Q>0$ and consequently $P \geq 3$ because $D=P^{2}-4 Q>0$. Then $\alpha \geq 2$ and $\beta>0$ because $\beta=\frac{P-\sqrt{P^{2}-4 Q}}{2}$. Therefore,

$$
\alpha^{i}(\alpha-1)>\beta^{i}(1-\beta) .
$$

Indeed, if $0<\beta \leq 1$, then $\beta^{i} \leq 1$ and $0 \leq 1-\beta<1$ which implies $0 \leq \beta^{i}(1-\beta)<$ 1. Moreover, $\alpha^{i}(\alpha-1) \geq 1$. Hence

$$
\alpha^{i}(\alpha-1)>\beta^{i}(1-\beta)
$$

which is evidently verified when $\beta>1$. Therefore $\alpha^{i+1}+\beta^{i+1}>\alpha^{i}+\beta^{i}$; i.e. $V_{i+1}>V_{i}$ and the proof is complete.

Lemma 2.6 If $(P, Q)$ is not standard then $\frac{\left|V_{2}\right|}{\left|V_{1}\right|} \approx+\infty$.

Proof We have two cases.

(A) $P \approx+\infty$. We divide the proof of A) further into the following cases.

(1) $Q$ standard. Then $\frac{V_{2}}{V_{1}}=\frac{P^{2}-2 Q}{P} \approx+\infty$.

(2) $Q \approx-\infty$. Then $\frac{V_{2}}{V_{1}}=\frac{P^{2}-2 Q}{P} \approx+\infty$.

(3) $Q \approx+\infty$. Suppose that $\frac{V_{2}}{V_{1}}=l$ with $l$ being limited. Then $\frac{P^{2}-2 Q}{P}=l>0$ $\left(P^{2}-2 Q>0\right.$ because $\left.D>0\right)$. Hence $P^{2}-P l=2 Q$ which implies $Q=\frac{1}{2}\left(P^{2}-P l\right)$. Then

$$
D=P^{2}-4 Q=P^{2}-2\left(P^{2}-P l\right)=-P^{2}+2 P l
$$

which means that $D<0$ and this is a contradiction. Then $\frac{V_{2}}{V_{1}} \approx+\infty$.

(B) $P$ standard. In this case $Q \approx-\infty$ and we show easily that $\frac{V_{2}}{V_{1}} \approx+\infty$. This finishes the proof.

Lemma 2.7 $n$ may be written according to one and only one of the following forms. 
(i) $n=p \approx+\infty$ is a prime.

(ii) $n=2^{s} p$, where $s \geq 1$ is a limited and $p \approx+\infty$ is a prime.

(iii) $n=n_{1} n_{2}$ where one of $n_{1}, n_{2}$ is odd greater than or equal to 3 , the other is unlimited.

(iv) $n=2^{\omega+1}$ with $\omega \approx+\infty$.

Proof It is well-known that $n$ must be written uniquely as $t_{1}^{\alpha_{1}} t_{2}^{\alpha_{2}} \ldots t_{r}^{\alpha_{r}}$, where $2 \leq$ $t_{1}<t_{2}<. .<t_{r}$ are prime numbers, $\alpha_{i} \geq 1(i=1,2, \ldots, r)$. Two cases arise.

(1) $r=1$. Either $t_{1}=2$, in which case $n$ is of the fourth form, or $t_{1}>2$ which implies that $n$ is of the first form if $\alpha_{1}=1$ because in this case $n=t_{1}$, otherwise $\left(\alpha_{1}>1\right)$, $n=t_{1}^{\alpha_{1}}=t_{1} t_{1}^{\alpha_{1}-1}$ which shows that $n$ is of the third form because the quantities $t_{1}$ and $t_{1}^{\alpha_{1}-1}$ are both odd and obviously at least one of them is unlimited.

(2) $r>1$. Here two subcases arise.

a) $t_{1}=2$.

If $\alpha_{1}$ is limited, then we divide the proof into two further cases.

(a1) $\alpha_{2}=1$. If $r=2$, then $n=2^{\alpha_{1}} t_{2}$ which shows that $t_{2}$ is unlimited and consequently $n$ is of the second form, otherwise (i.e. $r>2$ ) the fact that $n=2^{\alpha_{1}} t_{2} t_{3}^{\alpha_{3}} \ldots t_{r}^{\alpha_{r}}$ where $t_{2}$ is odd and $2^{\alpha_{1}} t_{3}^{\alpha_{3}} \ldots t_{r}^{\alpha_{r}} \approx+\infty$ because $2^{\alpha_{1}} t_{3}^{\alpha_{3}} \ldots t_{r}^{\alpha_{r}}>t_{2}$ and the product $t_{2} \cdot 2^{\alpha_{1}} t_{3}^{\alpha_{3}} \ldots t_{r}^{\alpha_{r}}=n \approx+\infty$, shows that $n$ is of the third form.

(a2) $\alpha_{2}>1$. In this case $n$ is of the third form because $n=2^{\alpha_{1}} t_{2}^{\alpha_{2}} t_{3}^{\alpha_{3}} \ldots t_{r}^{\alpha_{r}}=$ $t_{2} .2^{\alpha_{1}} t_{2}^{\alpha_{2}-1} t_{3}^{\alpha_{3}} \ldots t_{r}^{\alpha_{r}}$ where $t_{2}$ is odd and by the same reasoning as (a1) above, $r>2$ ) $2^{\alpha_{1}} t_{2}^{\alpha_{2}-1} t_{3}^{\alpha_{3}} \ldots t_{r}^{\alpha_{r}} \approx+\infty$.

If $\alpha_{1}$ is unlimited, then the fact that $n=2^{\alpha_{1}} t_{2}^{\alpha_{2}} t_{3}^{\alpha_{3}} \ldots t_{r}^{\alpha_{r}}=t_{2} .2^{\alpha_{1}} t_{2}^{\alpha_{2}-1} t_{3}^{\alpha_{3}} \ldots t_{r}^{\alpha_{r}}$, where $t_{2}$ is odd and $2^{\alpha_{1}} t_{2}^{\alpha_{2}-1} t_{3}^{\alpha_{3}} \ldots t_{r}^{\alpha_{r}} \approx+\infty$ permits us to conclude that $n$ is of the third form.

(b) $t_{1}>2$. Here also, using the same reasoning as above and the fact that $n=t_{1}^{\alpha_{1}} t_{2}^{\alpha_{2}} \ldots t_{r}^{\alpha_{r}}=t_{1} t_{1}^{\alpha_{1}-1} t_{2}^{\alpha_{2}} \ldots t_{r}^{\alpha_{r}} \approx+\infty$, we conclude that $n$ is of the third form.

We now prove that $n$ cannot be written simultaneously according to two of the above indicated forms. Indeed, we prove this for the second and the third form (the other cases are trivial). Suppose that $n=2^{s} p$ where $s \geq 1$ is a limited and $p \approx+\infty$ is a prime and also $n=n_{1} n_{2}$, where for example $n_{1}$ is odd greater than or equal to 3 and $n_{2}$ is unlimited. Since the decomposition of $n$ in prime factors is unique, then $n_{1}=p$, $n_{2}=2^{s}$ which is contradictory because $n_{2}$ is unlimited and cannot be equal to $2^{s}$. 


\section{Proof of Theorem 2.1.}

\subsection{Proof for $U_{n}$.}

We consider the following two subcases.

(I) $n$ is a prime. By $(1-5), U_{n}=\left(\frac{D}{n}\right) \bmod (n)$. Then $U_{n}=u+k n$, where $u \in\{-1,0,+1\}$. Since $\left|U_{n}\right|$ is, according to Lemma 2.3, of the form $\omega n$ with $\omega$ is an unlimited real number, the integer $k$ must be unlimited. Consequently the proof is finished for this case.

(II) $n$ is a composite i.e. $n=n_{1} n_{2}$, where $n_{1} \geq n_{2}>1$. By (1-6), $U_{n}=C U_{n_{1}}$, where $C$ is an integer which is, according to Lemma 2.4, unlimited. On the other hand since $n_{1} \approx+\infty$, then by Lemma 2.5, $U_{n_{1}}$ is unlimited. Thus the proof is finished for $U_{n}$.

\subsection{Proof for $V_{n}$}

By Lemma 2.7, we need to consider the following four cases.

(I) $n=p \approx+\infty$ is a prime. We have two subcases to consider.

(a) $P$ limited. By $(1-5), V_{p}=P \bmod (p)$; i.e. $V_{p}=P+k p$. Since $P$ is limited, $k$ must be, according to Lemma 2.3, unlimited.

(b) $P$ unlimited. According to (1-6), $V_{1} \mid V_{p}$; i.e $V_{p}=V_{1} N$. By Lemma 2.5, we have

$$
\left|V_{2}\right|<\left|V_{3}\right|<\ldots<\left|V_{n}\right|<\ldots .
$$

By Lemma 2.6, $\frac{\left|V_{2}\right|}{\left|V_{1}\right|} \approx+\infty$ which implies from $\frac{\left|V_{2}\right|}{\left|V_{1}\right|}<\frac{\left|V_{p}\right|}{\left|V_{1}\right|}$ that $\frac{\left|V_{p}\right|}{\left|V_{1}\right|} \approx+\infty$ and then $N$ is unlimited. Thus the proof is finished for this case because $V_{1}=P$ and $|P| \approx+\infty$.

(II) $n=2^{s} p$, where $s \geq 1$ is a limited and $p \approx+\infty$ is a prime.

(a) $P$ and $Q$ are both limited. For $s \geq 1$ define the formula

$A(s) \equiv$ "For $n$ of the form $2^{s} p, V_{n}$ may be written as $g_{1}+g_{2} p$ where $g_{1}\left(\right.$ resp. $\left.g_{2}\right)$ is a limited (resp. is an unlimited) integer".

We have $A$ (1). Indeed, let $n=2 p$; by (1-4)

$$
V_{n}=V_{2 p}=\left(V_{p}\right)^{2}-2 Q^{p} \text {. }
$$


Applying (1-5) and (1-8) yields $V_{2 p}=(P+k p)^{2}-2(Q+l p)$. Hence

$$
\begin{aligned}
V_{n} & =V_{2 p}=P^{2}+2 P k p+k^{2} p^{2}-2 Q-2 l p \\
& =P^{2}-2 Q+\left(2 P k+k^{2} p-2 l\right) p .
\end{aligned}
$$

If $g_{1}=P^{2}-2 Q$ and $g_{2}=2 P k+k^{2} p-2 l$, then $g_{1}$ is limited and, according to Lemma 2.3, $g_{2}$ is unlimited. Consequently, $A(1)$.

Suppose $A(s)$ for $s \geq 1$ a limited integer and prove $A(s+1)$. Indeed, by (1-4)

$$
V_{2^{s+1} p}=V_{2\left(2^{s} p\right)}=\left(V_{2^{s} p}\right)^{2}-2 Q^{2^{s} p} .
$$

From $A(s)$ (resp. (1-8)) we have $V_{2^{s}}=g_{1}+g_{2} p$, where $g_{1}$ is limited and $g_{2}$ is unlimited ( resp. $Q^{2^{s} p}=\left(Q^{2^{s}}\right)^{p}=Q^{2^{s}}+f p$ with $f$ an integer). Replacing by these values, we get

$$
\begin{aligned}
V_{2^{s+1} p} & =\left(V_{2^{s} p}\right)^{2}-2 Q^{2^{s} p} \\
& =\left(g_{1}+g_{2} p\right)^{2}-2\left(Q^{2^{s}}+f p\right) \\
& =g_{1}^{2}-2 Q^{2^{s}}+\bar{f} p,
\end{aligned}
$$

where $\bar{f}=2 g_{1} g_{2}+g_{2}^{2} p-2 f$. Since $g_{1}, Q$ and $s$ are limited, then $g_{1}^{2}-2 Q^{2^{s}}$ is limited; the integer $\bar{f}$, according to Lemma 2.3, must be unlimited. Consequently, $A(s+1)$.

Then by (1-9),

$$
\forall^{s t} s \geq 1 \quad A(s)
$$

(b) $P$ or $Q$ is unlimited. By (1-6), $V_{2^{s}} \mid V_{2^{s} p}$, i.e. $V_{2^{s}}=V_{2^{s}} c$ with $c$ being an integer. By Lemma 2.4, $c$ is unlimited. By Lemma 2.6, $\left|V_{2}\right| \approx+\infty$ and by Lemma 2.5, $\left|V_{2}\right|<\left|V_{3}\right|<\left|V_{4}\right|<\ldots$. Hence $V_{2^{s}}$ is unlimited. This completes the proof for this case.

(III) $n=n_{1} n_{2}$, where one of $n_{1}, n_{2}$ is odd greater than or equal to 3 , the other is unlimited.

Suppose $n_{1} \geq 3$ is odd and $n_{2} \approx+\infty$. Then

$$
V_{n_{1} n_{2}}=V_{n_{2}} C,
$$

where by (1-6) $C$ is an integer which is, according to Lemma 2.4., unlimited. Since $n_{2} \approx+\infty$, then by Lemma $2.5 V_{n_{2}}$ is unlimited. This finishes the proof for this case.

(IV) $n=2^{\omega+1}$ with $\omega \approx+\infty$.

(a) $Q$ is even $\left(Q=2 t, t \in \mathbb{Z}^{*}\right)$. By (1-4), we have

$$
V_{n}=V_{2^{\omega+1}}=V_{2.2^{\omega}}=V_{2^{\omega}}^{2}-2 Q^{2^{\omega}} .
$$


Applying the fact that $2^{\omega}=2.2^{\omega-1}$ and (1-4) yield

$$
V_{2^{\omega}}=V_{2.2^{\omega-1}}=V_{2^{\omega-1}}^{2}-2 Q^{2^{\omega-1}}
$$

which, when substituted in $V_{n}=V_{2 \omega}^{2}-2 Q^{2^{\omega}}$, gives

$$
\begin{aligned}
V_{n} & =V_{2^{\omega+1}}=\left(V_{2^{\omega-1}}^{2}-2 Q^{2^{\omega-1}}\right)^{2}-2 Q^{2^{\omega}} \\
& =\left(V_{2^{\omega-1}}\right)^{4} \bmod \left(Q^{2^{\omega-1}}\right) .
\end{aligned}
$$

Similarly, the fact that $V_{2^{\omega-1}}=V_{2.2^{\omega-2}}$ and (1-4) give

$$
V_{n}=V_{2^{\omega+1}}=\left(V_{2^{\omega-2}}\right)^{8} \bmod \left(Q^{2^{\omega-2}}\right)
$$

and so on.

Let $f \approx+\infty$ be an integer such that $\omega-f \approx+\infty$. The previous process permits to write

$$
V_{n}=V_{2^{\omega+1}}=\left(V_{2^{\omega-f}}\right)^{2^{f+1}} \bmod \left(Q^{2^{\omega-f}}\right),
$$

where $V_{2^{\omega-f}}$ is unlimited.

Now, if $V_{2^{\omega-f}}$ is even then $V_{2^{\omega+1}}=2^{\gamma} t$, where $\gamma=\min \left(2^{f+1}, 2^{\omega-f}\right) \approx+\infty$ and $t$ is an integer. This shows that

$$
V_{n}=V_{2^{\omega+1}}=2^{\gamma_{1}} 2^{\gamma_{2}} t
$$

where $\gamma_{1}$ and $\gamma_{2}$ are two unlimited integers satisfying $\gamma_{1}+\gamma_{2}=\gamma$. Otherwise (i.e. $V_{2^{\omega-f}}$ is odd), we have

$$
V_{n}-1=\left[\left(V_{2^{\omega-f}}\right)^{2^{f+1}}-1\right]+k Q^{2^{\omega-f}} .
$$

Since $\left(V_{2^{\omega-f}}\right)^{2^{f+1}}-1$ is a difference between squares, then

$$
V_{n}-1=\left[\left(V_{2^{\omega-f}}\right)^{2^{f}}-1\right]\left[\left(V_{2^{\omega-f}}\right)^{2^{f}}+1\right]+k Q^{2^{\omega-f}} .
$$

By the same reasoning about the difference $\left(V_{2^{\omega-f}}\right)^{2^{f}}-1$

$$
V_{n}-1=\left[\left(V_{2^{\omega-f}}\right)^{2^{f-1}}-1\right]\left[\left(V_{2^{\omega-f}}\right)^{2^{f-1}}+1\right]\left[\left(V_{2^{\omega-f}}\right)^{2^{f}}+1\right]+k Q^{2^{\omega-f}}
$$

and so on. Thus we can write $V_{n}-1$ as

$$
\begin{gathered}
V_{n}-1=\left[\left(V_{2^{\omega-f}}\right)^{2^{f-t}}-1\right]\left[\left(V_{2^{\omega-f}}\right)^{2^{f-t}}+1\right]\left[\left(V_{2^{\omega-f}}\right)^{2^{f-(t-1)}}+1\right]+\ldots \\
+\left[\left(V_{2^{\omega-f}}\right)^{2^{f-1}}+1\right]\left[\left(V_{2^{\omega-f}}\right)^{2^{f}}+1\right]+k Q^{2^{\omega-f}},
\end{gathered}
$$


where $t$ is an integer satisfying $1 \leq t<f$. Now choose $t_{0} \approx+\infty$ such that $t_{0}<f$ and $t_{0}+2<2^{\omega-f}$. This is possible because, from the fact that $\min \left(f, 2^{\omega-f}\right) \approx$ $+\infty$, we can choose an integer $s \approx+\infty$ such that $s \leq \min \left(f, 2^{\omega-f}\right)$ and take thereafter $t_{0}=s-3$. Since $Q^{2^{\omega-f}}$ contains the factor $2^{2^{\omega-f}}$ and the product $\left[\left(V_{2^{\omega-f}}\right)^{2^{f-t_{0}}}-1\right] \prod_{i=0}^{t_{0}}\left[\left(V_{2^{\omega-f}}\right)^{2^{f-i}}+1\right]$ contains $2^{k}$ with $k \geq t_{0}+2$, then
$V_{n}-1=2^{t_{0}+2} N$,

where $N$ is an integer. Therefore

$$
V_{n}-1=V_{2^{\omega+1}}-1=2^{t_{1}} 2^{t_{2}} N,
$$

where $t_{1}$ and $t_{2}$ are two unlimited integers satisfying $t_{1}+t_{2}=t_{0}+2$.

(b) $Q$ is odd $(Q=2 t+1, t \in \mathbb{Z})$. We put $n_{o}=2^{\omega}$. If $Q= \pm 1$, then by (1-4)

$$
\begin{aligned}
V_{n}=V_{2 n_{0}} & =\left(V_{n_{0}}\right)^{2}-2 Q^{n_{0}} \\
& =\left(V_{n_{0}}\right)^{2}-2
\end{aligned}
$$

because $n_{0}$ is even. $V_{n_{0}}$ is, by Lemma 2.3, unlimited. Otherwise (i.e. $Q \neq \pm 1$ ) we divide the proof into the following cases.

(1) $P$ is even. By (1-3) and the induction, we show easily that each $V_{l}(l \geq 0)$ is even. Moreover $V_{2} \neq 2$, because otherwise $P^{2}-2 Q=2$ which implies $D=$ $P^{2}-4 Q=2-2 Q$. The fact that $D>0$ implies that $2-2 Q>0$ i.e. $Q<0$ (because $Q \in \mathbb{Z}^{*}$ ) and this contradicts $P^{2}-2 Q=2$. In the same way $V_{2} \neq-2$.

Now, we prove that $V_{n}-2$ is the product of two unlimited integers. Indeed, by (1-4)

$$
V_{n}=V_{2^{\omega+1}}=V_{2 n_{0}}=V_{n_{0}}^{2}-2 Q^{n_{0}} \text {. }
$$

Then

$$
\begin{aligned}
V_{n}-2 & =V_{n_{0}}^{2}-4-2 Q^{n_{0}}+2 \\
& =\left(V_{n_{0}}-2\right)\left(V_{n_{0}}+2\right)-2\left(Q^{n_{0}}-1\right)
\end{aligned}
$$

which implies

$$
V_{n}-2=\left(V_{n_{0}}-2\right)\left(V_{n_{0}}+2\right)-2\left(Q^{n_{0} / 2}-1\right)\left(Q^{n_{0} / 2}+1\right) .
$$

Because $n_{0}$ is divisible by 2 , then applying (1-4) shows that $V_{n_{0}}-2$ can be written as

$$
V_{n_{0}}-2=V_{2\left(n_{0} / 2\right)}-2=V_{\left(n_{0} / 2\right)}^{2}-4-2\left(Q^{n_{0} / 2}-1\right)
$$

which, when substituted in (3-4), gives

$$
V_{n}-2=\left[V_{\left(n_{0} / 2\right)}^{2}-4-2\left(Q^{n_{0} / 2}-1\right)\right]\left(V_{n_{0}}+2\right)-2\left(Q^{n_{0} / 2}-1\right)\left(Q^{n_{0} / 2}+1\right) \text {. }
$$


Hence,

$$
\begin{aligned}
V_{n}-2= & \left(V_{\left(n_{0} / 2\right)}-2\right)\left(V_{\left(n_{0} / 2\right)}+2\right)\left(V_{n_{0}}+2\right) \\
& -2\left(Q^{n_{0} / 4}-1\right)\left(Q^{n_{0} / 4}+1\right)\left(V_{n_{0}}+2\right) \\
& -2\left(Q^{n_{0} / 4}-1\right)\left(Q^{n_{0} / 4}+1\right)\left(Q^{n_{0} / 2}+1\right) .
\end{aligned}
$$

Because $n_{0} / 2$ is divisible by 2 , then applying (1-4) shows that $V_{n_{0} / 2}-2$ can be written as

$$
\begin{aligned}
V_{\left(n_{0} / 2\right)}-2 & =V_{\left(n_{0} / 4\right)}^{2}-2 Q^{n_{0} / 4}-4+2 \\
& =\left(V_{\left(n_{0} / 4\right)}^{2}-4\right)-2\left(Q^{n_{0} / 4}-1\right)
\end{aligned}
$$

which, when substituted in (3-5), gives

$$
\begin{aligned}
V_{2 n_{0}}-2= & \left(V_{\left(n_{0} / 4\right)}-2\right)\left(V_{\left(n_{0} / 4\right)}+2\right)\left(V_{\left(n_{0} / 2\right)}+2\right)\left(V_{n_{0}}+2\right) \\
& -2\left(Q^{n_{0} / 8}-1\right)\left(Q^{n_{0} / 8}+1\right)\left(V_{\left(n_{0} / 2\right)}+2\right)\left(V_{n_{0}}+2\right) \\
& -2\left(Q^{n_{0} / 8}-1\right)\left(Q^{n_{0} / 8}+1\right)\left(Q^{n_{0} / 4}+1\right)\left(V_{n_{0}}+2\right) \\
& -2\left(Q^{n_{0} / 8}-1\right)\left(Q^{n_{0} / 8}+1\right)\left(Q^{n_{0} / 4}+1\right)\left(Q^{n_{0} / 2}+1\right)
\end{aligned}
$$

and so on.

So the process of applying (1-4) and putting the difference between squares as a product of two factors, yields by induction

$$
\begin{aligned}
& V_{n}-2=V_{2 n_{0}}-2 \\
& =\left(V_{n_{0} / 2^{i-1}}-2\right)\left(V_{n_{0} / 2^{i-1}}+2\right) \ldots\left(V_{n_{0} / 2}+2\right)\left(V_{n_{0}}+2\right) \\
& \quad-2\left(Q^{n_{0} / 2^{i}}-1\right)\left(Q^{n_{0} / 2^{i}}+1\right)\left(V_{n_{0} / 2^{i-2}}+2\right) \ldots\left(V_{n_{0} / 2}+2\right)\left(V_{n_{0}}+2\right) \\
& \quad-2\left(Q^{n_{0} / 2^{i}}-1\right)\left(Q^{n_{0} / 2^{i}}+1\right)\left(Q^{n_{0} / 2^{i-1}}+1\right)\left(V_{n_{0} / 2^{i-3}}+2\right) \ldots\left(V_{n_{0}}+2\right) \\
& \quad-2\left(Q^{n_{0} / 2^{i}}-1\right)\left(Q^{n_{0} / 2^{i}}+1\right)\left(Q^{n_{0} / 2^{i-1}}+1\right)\left(Q^{n_{0} / 2^{i-2}}+1\right)\left(V_{n_{0} / 2^{i-4}}+2\right) \ldots\left(V_{n_{0}}+2\right) \\
& \quad \ldots \\
& \quad-2\left(Q^{n_{0} / 2^{i}}-1\right)\left(Q^{n_{0} / 2^{i}}+1\right)\left(Q^{n_{0} / 2^{i-1}}+1\right) \ldots\left(Q^{n_{0} / 2^{2}}+1\right)\left(V_{n_{0}}+2\right) \\
& \quad-2\left(Q^{n_{0} / 2^{i}}-1\right)\left(Q^{n_{0} / 2^{i}}+1\right)\left(Q^{n_{0} / 2^{i-1}}+1\right) \ldots\left(Q^{n_{0} / 2^{2}}+1\right)\left(Q^{n_{0} / 2}+1\right),
\end{aligned}
$$

where $1 \leq i \leq \omega$. In this formula, if we replace $i$ by 1 we recover (3-4), by 2 we recover (3-5), etc. 
We take $i_{0} \approx+\infty$ such that $\frac{n_{0}}{2^{i_{0}}} \geq 1$. We show that $V_{n}-2$ is of the form $2^{i_{0}+1} t$, where $t$ is an integer. Indeed, each element $V_{n_{0} / 2^{j}}\left(0 \leq j \leq i_{0}-1\right)$ is even and, according to Lemma 2.5, different from \pm 2 giving the fact that $V_{2}$ is different from these values. On the other hand $Q$ is odd and different from \pm 1 . Hence the formula (3-7) is the sum of $i_{0}+1$ terms, where each term is the product of $i_{0}+1$ non-zero even integers. Therefore

$$
V_{n}-2=2^{i_{0}+1} t=2^{t_{1}} 2^{t_{2}} t
$$

where $t_{1}$ and $t_{2}$ are two unlimited integers satisfying $t_{1}+t_{2}=i_{0}+1$ and $t$ is an integer.

(2) $P$ is odd. We prove by induction that $V_{2^{l}}(l \geq 1)$ is odd. Indeed, $V_{2^{1}}=$ $P^{2}-2 Q$ this shows that $V_{2}$ is odd. Now suppose that $V_{2^{l}}$ is odd with $l \geq 1$. Then $V_{2^{l+1}}=\left(V_{2^{l}}\right)^{2}-2 Q^{2^{l}}$ so $V_{2^{l+1}}$ is also odd. On the other hand $V_{2} \neq 1$, otherwise $P^{2}-2 Q=1$ then the fact that $D=P^{2}-4 Q=1-2 Q>0$ implies that $Q<0$ and this contradicts $P^{2}-2 Q=1$. In the same way $V_{2} \neq-1$.

By $(1-4)$

$$
V_{n}=V_{2^{\omega+1}}=V_{2 n_{0}}=V_{n_{0}}^{2}-2 Q^{n_{0}}
$$

Then

$$
V_{n}+1=V_{n_{0}}^{2}-1+2-2 Q^{n_{0}}=\left(V_{n_{0}}-1\right)\left(V_{n_{0}}+1\right)+2\left(1-Q^{n_{0}}\right)
$$

So

$$
V_{n}+1=\left(V_{n_{0}}+1\right)\left(V_{n_{0}}-1\right)+2\left(1-Q^{n_{0} / 2}\right)\left(1+Q^{n_{0} / 2}\right) .
$$

By $(1-4)$

$$
\begin{aligned}
V_{n_{0}}+1 & =V_{2\left(n_{0} / 2\right)}+1 \\
& =\left[V_{\left(n_{0} / 2\right)}^{2}-1+2-2 Q^{\left(n_{0} / 2\right)}\right] \\
& =\left[\left(V_{\left(n_{0} / 2\right)}-1\right)\left(V_{\left(n_{0} / 2\right)}+1\right)+2\left(1-Q^{\left(n_{0} / 2\right)}\right)\right]
\end{aligned}
$$

which, when substituted in (3-8), gives

$$
\begin{gathered}
V_{n}+1=\left[\left(V_{\left(n_{0} / 2\right)}-1\right)\left(V_{\left(n_{0} / 2\right)}+1\right)+2\left(1-Q^{\left(n_{0} / 2\right)}\right)\right]\left(V_{n_{0}}-1\right) \\
+2\left(1-Q^{n_{0} / 2}\right)\left(1+Q^{n_{0} / 2}\right) \\
=\left(V_{\left(n_{0} / 2\right)}-1\right)\left(V_{\left(n_{0} / 2\right)}+1\right)\left(V_{n_{0}}-1\right)+2\left(1-Q^{\left(n_{0} / 2\right)}\right)\left(V_{n_{0}}-1\right) \\
+2\left(1-Q^{\left(n_{0} / 2\right)}\right)\left(1+Q^{\left(n_{0} / 2\right)}\right)
\end{gathered}
$$


Then

$$
\begin{aligned}
V_{n}+1= & \left(V_{\left(n_{0} / 2\right)}-1\right)\left(V_{\left(n_{0} / 2\right)}+1\right)\left(V_{n_{0}}-1\right) \\
& +2\left(1-Q^{\left(n_{0} / 4\right)}\right)\left(1+Q^{\left(n_{0} / 4\right)}\right)\left(V_{n_{0}}-1\right) \\
& +2\left(1-Q^{\left(n_{0} / 4\right)}\right)\left(1+Q^{\left(n_{0} / 4\right)}\right)\left(1+Q^{\left(n_{0} / 2\right)}\right)
\end{aligned}
$$

Again, setting $\frac{n_{0}}{2}=2 \cdot \frac{n_{0}}{4}$ and calculating by (1-4) an expression for $V_{\left(n_{0} / 2\right)}+1$ and substituting in (3-9), we get another formula for $V_{n}+1$, and so on.

So this process yields by induction

$V_{n}+1=V_{2^{\omega+1}}+1$

$=\left(V_{n_{0} / 2^{i}}+1\right)\left(V_{n_{0} / 2^{i}}-1\right)\left(V_{n_{0} / 2^{i-1}}-1\right) \ldots\left(V_{n_{0} / 2}-1\right)\left(V_{n_{0}}-1\right)$

$+2\left(1-Q^{n_{0} / 2^{i+1}}\right)\left(1+Q^{n_{0} / 2^{i+1}}\right)\left(V_{n_{0} / 2^{i-1}}-1\right)\left(V_{n_{0} / 2^{i-2}}-1\right) \ldots\left(V_{n_{0}}-1\right)$

$+2\left(1-Q^{n_{0} / 2^{i+1}}\right)\left(1+Q^{n_{0} / 2^{i+1}}\right)\left(1+Q^{n_{0} / 2^{i}}\right)\left(V_{n_{0} / 2^{i-2}}-1\right) \ldots\left(V_{n_{0}}-1\right)$

$+2\left(1-Q^{n_{0} / 2^{i+1}}\right)\left(1+Q^{n_{0} / 2^{i+1}}\right)\left(1+Q^{n_{0} / 2^{i}}\right)\left(1+Q^{n_{0} / 2^{i-1}}\right)\left(V_{n_{0} / 2^{i-3}}-1\right) . .\left(V_{n_{0}}-1\right)+$ $\cdots$

$+2\left(1-Q^{n_{0} / 2^{i+1}}\right)\left(1+Q^{n_{0} / 2^{i+1}}\right)\left(1+Q^{n_{0} / 2^{i}}\right)\left(1+Q^{n_{0} / 2^{i-1}}\right) \cdots\left(1+Q^{n_{0} / 2^{2}}\right)\left(V_{n_{0}}-1\right)$

$+2\left(1-Q^{n_{0} / 2^{i+1}}\right)\left(1+Q^{n_{0} / 2^{i+1}}\right)\left(1+Q^{n_{0} / 2^{i}}\right)\left(1+Q^{n_{0} / 2^{i-1}}\right) \ldots\left(1+Q^{n_{0} / 2}\right)$,

where $0 \leq i \leq \omega-1$. In this formula, if we replace $i$ by 0 we recover (3-8), by 1 we recover (3-9) etc... .

We take $i_{0} \approx+\infty$ such that $\frac{n_{0}}{2^{i_{0}}} \geq 2$. We show that $V_{n}+1$ is of the form $2^{i_{0}+2} t$, where $t$ is an integer. Indeed, each element $V_{n_{0} / 2^{j}}\left(0 \leq j \leq i_{0}\right)$ is odd and, according to Lemma 2.5, different from \pm 1 giving the fact that $V_{2}$ is different from these values. On the other hand $Q$ is odd and different from \pm 1 . Hence the formula (3-10) is the sum of $i_{0}+2$ terms, where each term is the product of $i_{0}+2$ non-zero even integers. From this

$$
V_{n}+1=V_{2 n_{0}}+1=2^{t_{1}} 2^{t_{2}} t,
$$

where $t_{1}$ and $t_{2}$ are two unlimited integers satisfying $t_{1}+t_{2}=i_{0}+2$ and $t$ is an integer. This finishes the proof of this case and therefore the theorem. 


\section{In classical terms}

In order to find the classical equivalence of our result we use the reduction algorithm of external formulas of Nelson [5]. We denote the standard set

$$
\left\{(P, Q) \in\left(\mathbb{Z}^{*}\right)^{2}: P^{2}-4 Q>0\right\}
$$

by $H$. Theorem 2.1 may be written as

$$
\begin{aligned}
& \forall n \forall(P, Q) \\
& {\left[\begin{array}{r}
\left(\forall^{s t} t: n \geq t\right) \Rightarrow \exists^{s t}(U, V) \exists\left(l_{1}, l_{2}, l_{1}^{\prime}, l_{2}^{\prime}\right) \forall^{s t} i \in \mathbb{N} \\
\left(U_{n}=U+l_{1} l_{2}, V_{n}=V+l_{1}^{\prime} l_{2}^{\prime} \& \min \left(\left|l_{1}\right|,\left|l_{2}\right|,\left|l_{1}^{\prime}\right|,\left|l_{2}^{\prime}\right|\right)>i\right)
\end{array}\right],}
\end{aligned}
$$

where $n, t, i$ range over $\mathbb{N},(P, Q)$ range over $H$ and $U, V, l_{1}, l_{2}, l_{1}^{\prime}, l_{2}^{\prime}$ range over $\mathbb{Z}$. Using idealization, this formula is equivalent to

$\forall n \forall(P, Q)$

$$
\left[\begin{array}{rl}
\left(\forall^{s t} t: n \geq t\right) \Rightarrow \exists^{s t}(U, V) \forall^{s t f i n i} I \exists\left(l_{1}, l_{2}, l_{1}^{\prime}, l_{2}^{\prime}\right) \forall i \in I \\
\left(U_{n}=U+l_{1} l_{2}, V_{n}=V+l_{1}^{\prime} l_{2}^{\prime} \& \min \left(\left|l_{1}\right|,\left|l_{2}\right|,\left|l_{1}^{\prime}\right|,\left|l_{2}^{\prime}\right|\right)>i\right)
\end{array}\right],
$$

where $I$ belongs to $\mathcal{P}_{f}(\mathbb{N})$ the set of finite subsets of $\mathbb{N}$. This formula is equivalent to

$$
\begin{aligned}
& \forall(n,(P, Q)) \exists^{s t}(t,(U, V)) \forall^{s t f i n i} I \\
& {\left[\begin{array}{r}
(n \geq t) \Rightarrow \exists\left(l_{1}, l_{2}, l_{1}^{\prime}, l_{2}^{\prime}\right) \forall i \in I \\
\left.\left(U_{n}=U+l_{1} l_{2}, V_{n}=V+l_{1}^{\prime} l_{2}^{\prime} \& \min \left(\left|l_{1}\right|,\left|l_{2}\right|,\left|l_{1}^{\prime}\right|,\left|l_{2}^{\prime}\right|\right)>i\right)\right] .
\end{array}\right.}
\end{aligned}
$$

If we refer to $\forall \exists^{s t} \forall^{s t}$ in the lexicon cited in [5], the last formula is equivalent to

$$
\begin{aligned}
& \forall \widetilde{I} \exists^{f i n i} \mathcal{R} \forall(n,(P, Q)) \exists(t,(U, V)) \in \mathcal{R} \\
& \left.\qquad \begin{array}{l}
(n \geq t) \Rightarrow \exists\left(l_{1}, l_{2}, l_{1}^{\prime}, l_{2}^{\prime}\right) \forall i \in \widetilde{I}(t,(U, V)) \\
\left(U_{n}=U+l_{1} l_{2}, V_{n}=V+l_{1}^{\prime} l_{2}^{\prime} \& \min \left(\left|l_{1}\right|,\left|l_{2}\right|,\left|l_{1}^{\prime}\right|,\left|l_{2}^{\prime}\right|\right)>i\right)
\end{array}\right],
\end{aligned}
$$

where $\widetilde{I}$ is a mapping that associates with each $(t,(U, V)) \in \mathbb{N} \times \mathbb{Z}^{2}$ a finite subset $\widetilde{I}(t,(U, V)) \subset \mathbb{N}$. Now we prove that $(4-1)$ is equivalent to 


$$
\begin{gathered}
\forall \widetilde{I} \exists(T, W) \in \mathbb{N}^{2} \forall(n,(P, Q)) \exists(t,(U, V)) \in[0, T] \times[-W, W]^{2} \\
{\left[\begin{array}{c}
(n \geq t) \Rightarrow \exists\left(l_{1}, l_{2}, l_{1}^{\prime}, l_{2}^{\prime}\right) \forall i \in \widetilde{I}(t,(U, V)) \\
\left(U_{n}=U+l_{1} l_{2}, V_{n}=V+l_{1}^{\prime} l_{2}^{\prime} \& \min \left(\left|l_{1}\right|,\left|l_{2}\right|,\left|l_{1}^{\prime}\right|,\left|l_{2}^{\prime}\right|\right)>i\right)
\end{array}\right] .}
\end{gathered}
$$

Indeed, let $\widetilde{I}: \mathbb{N} \times \mathbb{Z}^{2} \rightarrow \mathcal{P}_{f}(\mathbb{N})$ be a set-valued mapping. Then according to (4-1) $\exists^{\text {fini }} \mathcal{R} \subset \mathbb{N} \times \mathbb{Z}^{2}$ and therefore there exist smaller integers $T$ and $W$ such that $\mathcal{R} \subset[0, T] \times[-W, W]^{2}$. Let $(n,(P, Q)) \in \mathbb{N} \times H$, by $(4-1) \exists(t,(U, V)) \in \mathcal{R} \subset$ $[0, T] \times[-W, W]^{2}$; that is, $(t,(U, V)) \in[0, T] \times[-W, W]^{2}$. Now if $n \geq t$, then $\exists\left(l_{1}, l_{2}, l_{1}^{\prime}, l_{2}^{\prime}\right) \forall i \in \widetilde{I}(t,(U, V))$

$$
U_{n}=U+l_{1} l_{2}, V_{n}=V+l_{1}^{\prime} l_{2}^{\prime} \& \min \left(\left|l_{1}\right|,\left|l_{2}\right|,\left|l_{1}^{\prime}\right|,\left|l_{2}^{\prime}\right|\right)>i \text { which shows that }
$$
$(4-1) \Rightarrow(4-2)$. For the converse one takes, $\mathcal{R}=[0, T] \times[-W, W]^{2}$.

Hence we have the following internal formulation of Theorem 2.1.

Proposition 4.1 For any set-valued mapping $\widetilde{I}: \mathbb{N} \times \mathbb{Z}^{2} \rightarrow P_{f}(\mathbb{N})$ there exists $(T, W) \in \mathbb{N}^{2}$ such that for all $(n,(P, Q)) \in \mathbb{N} \times H$ there exists $(t,(U, V)) \in[0, T] \times$ $[-W, W]^{2}$ such that if $n \geq t$, then $U_{n}$ (resp. $V_{n}$ ) differs by $U$ (resp. $V$ ) from a product of two integers whose absolute value is greater than or equal to $i$ for all $i \in \widetilde{I}(t,(U, V))$.

Below we formulate, from the proof of Theorem 2.1, two particular cases of that result, and give their reductions separately.

(1) $U_{n}$ differs from a product of two unlimited integers by an integer $U$ with $-1 \leq$ $U \leq 1$.

(2) If $n$ is not of the form $2^{s} p$ with $s \geq 0$ being a limited integer and $p \approx+\infty$ being a prime, then $V_{n}$ differs from a product of two unlimited integers by an integer $V$ with $-2 \leq V \leq 2$.

The reductions of these particular cases are as follows.

(1) The first is equivalent to

$$
\forall n \forall(P, Q)\left[\begin{array}{c}
\left(\forall^{s t} t: n \geq t\right) \Longrightarrow \exists\left(U, l_{1}, l_{2}\right) \forall^{s t} i \\
\left(U_{n}(P, Q)=U+l_{1} l_{2}:|U| \leq 1 \& \min \left(\left|l_{1}\right|,\left|l_{2}\right|\right) \geq i\right)
\end{array}\right],
$$

where $n, t, i \in \mathbb{N},\left(U, l_{1}, l_{2}\right) \in \mathbb{Z}^{3}$. By idealization and transfer the previous formula transforms, while remaining equivalent, to 
$\forall^{f i n i} I \exists^{\text {fini }} T \forall(n,(P, Q)) \exists t \in T$

$$
\left[\begin{array}{c}
n \geq t \Longrightarrow \exists\left(U, l_{1}, l_{2}\right) \forall i \in I \\
\left(U_{n}(P, Q)=U+l_{1} l_{2}:|U| \leq 1 \& \min \left(\left|l_{1}\right|,\left|l_{2}\right|\right) \geq i\right)
\end{array}\right],
$$

where $I$ and $T$ belong to the power set of $\mathbb{N}$. (4-3) is equivalent to $\forall i \in \mathbb{N} \exists \widetilde{T} \in \mathbb{N} \forall(n,(P, Q)) \in \mathbb{N} \times H$

$$
\left[\begin{array}{c}
n \geq \widetilde{T} \Longrightarrow \\
\exists\left(U, l_{1}, l_{2}\right) \quad\left(U_{n}(P, Q)=U+l_{1} l_{2} \text { with }|U| \leq 1 \& \min \left(\left|l_{1}\right|,\left|l_{2}\right|\right) \geq i\right)
\end{array}\right] .
$$

Indeed, let $i \in \mathbb{N}$. Then, according to (4-3), $\exists^{f i n i} T \subset \mathbb{N}$. Now put $\widetilde{T}=\max _{t \in T} t$. Now if $(n,(P, Q)) \in \mathbb{N} \times H$ such that $n \geq \widetilde{T}$, then $n \geq t, \forall t \in T$. Hence from (4-3) $\exists\left(U, l_{1}, l_{2}\right)$ with $U_{n}(P, Q)=U+l_{1} l_{2},|U| \leq 1$ and $\min \left(\left|l_{1}\right|,\left|l_{2}\right|\right) \geq$ i. Consequently (4-3) $\Longrightarrow(4-4)$.

Conversely, let $I$ be a finite subset of $\mathbb{N}$ and put $\bar{i}=\max _{i \in I} i$. For $\bar{i}$ there is, according to (4-4), $\widetilde{T} \in \mathbb{N}$. Consider $T=\{\widetilde{T}\}$ as a finite subset of $\mathbb{N}$. Now if $(n,(P, Q)) \in \mathbb{N} \times H$ such that $n \geq \widetilde{T}$, then $\exists\left(U, l_{1}, l_{2}\right)$ with $U_{n}(P, Q)=U+l_{1} l_{2}$ with $|U| \leq 1$ and $\min \left(\left|l_{1}\right|,\left|l_{2}\right|\right) \geq \bar{i}$; that is, $\min \left(\left|l_{1}\right|,\left|l_{2}\right|\right) \geq i \forall i \in I$. Hence (4-4) $\Longrightarrow$ (4-3) and consequently we have the following proposition.

Proposition 4.2 For any integer $i \in \mathbb{N}$ there exists an integer $\widetilde{T} \in \mathbb{N}$ such that for all $(n,(P, Q)) \in \mathbb{N} \times H$ satisfying $n \geq \widetilde{T}$, the term $U_{n}(P, Q)$ differs from a product of two integers whose absolute value is greater than or equal to $i$ by an integer $U$ with $-1 \leq U \leq 1$.

(2) The second particular case is equivalent to $\forall n \forall(P, Q)$

$$
\left[\begin{array}{c}
\forall^{s t}\left(t_{1}, t_{2}\right)\left(n \geq t_{1} \& \frac{n}{2^{t_{2}}} \notin \mathbb{P}\right) \Longrightarrow \\
\exists\left(V, l_{1}^{\prime}, l_{2}^{\prime}\right) \forall^{s t} i\left(V_{n}(P, Q)=V+l_{1}^{\prime} l_{2}^{\prime} \text { with } V \in\{0, \pm 1, \pm 2\} \text { and } \min \left(\left|l_{1}^{\prime}\right|,\left|l_{2}^{\prime}\right|\right) \geq i\right)
\end{array}\right],
$$

where $n, i \in \mathbb{N},\left(t_{1}, t_{2}\right) \in \mathbb{N}^{2},\left(V, l_{1}^{\prime}, l_{2}^{\prime}\right) \in\{0, \pm 1, \pm 2\} \times \mathbb{Z} \times \mathbb{Z}$. Using idealization and transfer the previous formula transforms, while remaining equivalent, to $\forall^{\text {fini } I} \exists^{\text {fini }} T \forall(n,(P, Q))$

$$
\left[\begin{array}{c}
\forall\left(t_{1}, t_{2}\right) \in T\left(n \geq t_{1} \& \frac{n}{2^{t_{2}}} \notin \mathbb{P}\right) \Longrightarrow \exists\left(V, l_{1}^{\prime}, l_{2}^{\prime}\right) \forall i \in I \\
\left(V_{n}(P, Q)=V+l_{1}^{\prime} l_{2}^{\prime}: V \in\{0, \pm 1, \pm 2\} \text { and } \min \left(\left|l_{1}^{\prime}\right|,\left|l_{2}^{\prime}\right|\right) \geq i\right)
\end{array}\right]
$$


where $I$ (resp. $T$ ) belongs to the power set of $\mathbb{N}$ (resp. $\mathbb{N}^{2}$ ). Then (4-5) is equivalent to

$\forall i \in \mathbb{N} \exists\left(T_{1}, T_{2}\right) \in \mathbb{N}^{2} \forall(n,(P, Q))$

$$
\left[\begin{array}{c}
\left(n \geq T_{1}, \frac{n}{2^{i}} \notin \mathbb{P}\left(i=0,1, . ., T_{2}\right)\right) \Longrightarrow \exists\left(V, l_{1}^{\prime}, l_{2}^{\prime}\right) \\
\left(V_{n}(P, Q)=V+l_{1}^{\prime} l_{2}^{\prime}: V \in\{0, \pm 1, \pm 2\} \text { and } \min \left(\left|l_{1}^{\prime}\right|,\left|l_{2}^{\prime}\right|\right) \geq i\right)
\end{array}\right] .
$$

Indeed, let $i \in \mathbb{N}$. According to (4-5), $\exists^{f i n i} T \subset \mathbb{N}^{2}$. Suppose

$T_{1}=\max \left\{t_{1}:\left(t_{1}, t_{2}\right) \in T\right\}$ and $T_{2}=\max \left\{t_{2}:\left(t_{1}, t_{2}\right) \in T\right\}$. Let $(n,(P, Q)) \in \mathbb{N} \times H$. Suppose that $n \geq T_{1}$ and $\frac{n}{2^{i}} \notin \mathbb{P}$ for $i=0,1, . ., T_{2}$. Then for all $\left(t_{1}, t_{2}\right) \in T, n \geq t_{1}$ and $\frac{n}{2^{t_{2}}} \notin \mathbb{P}$. Hence, according to (4-5), $\exists\left(V, l_{1}^{\prime}, l_{2}^{\prime}\right) V_{n}(P, Q)=V+l_{1}^{\prime} l_{2}^{\prime}$ with $V \in\{0, \pm 1, \pm 2\}$ and $\min \left(\left|l_{1}^{\prime}\right|,\left|l_{2}^{\prime}\right|\right) \geq i$. Consequently (4-5) $\Rightarrow(4-6)$.

Conversely, let $I$ be a finite subset of $\mathbb{N}$. Put $\bar{i}=\max _{i \in I} i$. Then for $\bar{i}$ there is, according to (4-6), $\left(T_{1}, T_{2}\right) \in \mathbb{N}^{2}$. Set $T=\left\{0,1, \ldots, T_{1}\right\} \times\left\{0,1, \ldots, T_{2}\right\}$, then $T$ is finite. Let $(n,(P, Q)) \in \mathbb{N} \times H$ and suppose that for all $\left(t_{1}, t_{2}\right) \in T, n \geq t_{1} \& \frac{n}{2^{t_{2}}} \notin \mathbb{P}$. Then $n \geq T_{1}$ and $\frac{n}{2^{i}} \notin \mathbb{P}\left(i=0,1, . ., T_{2}\right)$. Hence, according to (4-6), $\exists\left(V, l_{1}^{\prime}, l_{2}^{\prime}\right)$ such that $V_{n}(P, Q)=V+l_{1}^{\prime} l_{2}^{\prime}$ with $V \in\{0, \pm 1, \pm 2\}$ and $\min \left(\left|l_{1}^{\prime}\right|,\left|l_{2}^{\prime}\right|\right) \geq \bar{i}$; that is $\forall i \in I \min \left(\left|l_{1}^{\prime}\right|,\left|l_{2}^{\prime}\right|\right) \geq i$. Hence (4-6) $\Longrightarrow(4-5)$ and consequently we have the following proposition.

Proposition 4.3 For any integer $i \in \mathbb{N}$ there exists two integers $\left(T_{1}, T_{2}\right) \in \mathbb{N}^{2}$ such that for all $(n,(P, Q)) \in \mathbb{N} \times H$ satisfying $n \geq T_{1}$ and $\frac{n}{2^{i}} \notin \mathbb{P}$ for $i=0,1, . ., T_{2}$ the term $V_{n}(P, Q)$ differs from a product of two integers whose absolute value is greater than or equal to $i$, by an integer $V$ with $-2 \leq V \leq 2$.

General remark. In the classical literature concerning Lucas sequences, generally the studies are concerned with the terms $U_{k}$ and $V_{k}$ for $k$ belonging to a particular family of integers (see for example $[6,7]$ ). In this work we note from the previous propositions that the main result expresses a property of uniformity because the conclusion is valid for all $k$ beyond a certain rank.

Moreover, the ideas used in proofs here can also be used to deduce standard results. For example one sees without pain that Lemma 2.3 gives the size of $\left|U_{k}\right|$ and $\left|V_{k}\right|$ whereas Lemma 2.5 gives the growth of $\left|U_{k}\right|$ and $\left|V_{k}\right|$. In addition, the translation 
by the reduction algorithm of lemmas used previously, which is an operation that is not difficult, gives more classical results. But giving further details would increase the length of this paper.

Acknowledgment The author wishes to thank the referees and editor for their comments and suggestions for improving an earlier version of this paper.

\section{References}

[1] A. Balog, $p+a$ without large prime factors, Séminaire de théorie des nombres de Bordeaux , Exposé 31(1983-84).

[2] A. Boudaoud, La conjecture de Dickson et classes particulières d'entiers, Annales Mathématiques Blaise Pascal 13(2006), 103-109.

[3] Chen, M.J.R, On the representation of a large even integer as the sum of a prime and the product of at most two primes, Scientia Sinica 16(1973), 157 - 176.

[4] F. Diener et G. Reeb, Analyse Non-Standard, Hermann, Éditeurs des Sciences et des Arts, 1989.

[5] E. Nelson, Internal set theory: A new approach to nonstandard analysis, Bull. Amer. Math. Soc. 83(1977), 1165-1198; doi:10.1090/S0002-9904-1977-14398-X.

[6] Paulo Ribenboim, The Little Book of Big Primes, Springer-Verlag, 1991.

[7] Paulo Ribenboim, My numbers my friends, Springer-Verlag 2000.

[8] S. Salerno - A. Vitolo, $p+2$ with few and bounded prime factors, Analysis 11(1991), 129-148.

Department of Mathematics, University of Msila, Ichbilia BP 166 - 28000 - Msila, Algeria

boudaoudab@yahoo.fr

Received: 25 March 2008 Revised: 25 November 2008 